\title{
ON HARDY'S INEQUALITIES FOR THE SPECIAL HERMITE EXPANSIONS
}

\author{
JinSEN XiAO AND JiANXUN HE
}

Abstract. This article presents two types of Hardy's inequalities for the special Hermite expansions. The proofs are mainly based on an estimate of atomic functions deduced by the horizontal Taylor formula of the Heisenberg group. Mathematics subject classification (2010): 43A85, 44A12, 52A38. transform.

Keywords and phrases: Hardy's inequality, special Hermite expansions, Heisenberg group, Fourier

\section{REFERENCES}

[1] G. Arena, A. O. Caruso and A. Causa, Taylor formula on step two Carnot groups, Rev. Mat. Iberoamericana 26 (2010), 239-259.

[2] M. AssaL, Hardy's type inequality associated with the Hankel transform for over-critical exponent, Integr. Transf. Spec. F. 22 (2011), 45-50.

[3] R. Balasubramanian And R. Radha, Hardy-type inequalities for Hermite expansions, J. Inequal. Pure Appl. Math. 6 (2005), 1-4.

[4] J. J. Betancor, J. Dziubański and J. L. Torrea, On Hardy spaces associated with Bessel operators, J. Anal. Math. 107 (2009), 195-219.

[5] A. Bonfiglioli, Taylor formula for homogenous groups and applications, Math. Z. 262 (2009), $255-279$.

[6] P. L. Duren, Theory of $H^{p}$ spaces, Academic Press, New York, 1970.

[7] Y. KAnjin, Hardy's inequalities for Hermite and Laguerre expansions, Bull. London Math. Soc. 29 (1997), 331-337.

[8] Y. Kanjin, Hardy's inequalities for Hermite and Laguerre expansions revisited, J. Math. Soc. Japan. 63 (2011), 753-767.

[9] Y. KAnjin AND K. SATo, Hardy's inequality for Jacobi expansions, Math. Inequal. App. 7 (2004), 551-555.

[10] Z. LI, Y. YU AND Y. SHI, The Hardy inequality for Hermite expansions, J. Fourier Anal. Appl. 21 (2015), 267-280.

[11] C. C. LIN, Hörmander's $H^{p}$ multiplier theorem for the Heisenberg group, J. London Math. Soc. 67 (2003), 686-700.

[12] R. RADHA, Hardy-type inequalities, Taiwan. J. math. 4 (2000), 447-456.

[13] R. Radha And S. Thangavelu, Hardy's inequalities for Hermite and Laguerre expansions, Proc. Amer. Math. Soc. 132 (2004), 3525-3536.

[14] M. S ATAKE, Hardy's inequalities for Laguerre expansions, J. Math. Soc. Japan. 52 (2000), 17-24.

[15] K. SATO, Paley's inequality and Hardy's inequality for the Fourier-Bessel expansions, J. Nolinear Covex Anal. 6 (2005), 441-451.

[16] S. Thangavelu, On regularity of twisted spherical means and special Hermite expansion, Proc. Indian Acad. Sci. Math. Sci. 103 (1993), 303-320.

[17] S. Thangavelu, Lectures on Hermite and Laguerre Expansions, Math. Notes, no. 42, Princeton University Press, Princeton, NJ, 1993.

[18] S. Thangavelu, An introduction to the uncertainty principle: Hardy's theorem on Lie groups, Progress in Mathematics, 217. Birkhäuser, Boston, Basel, Berlin, 2003. 